\title{
El programa filosófico-político de Rousseau: el vínculo teórico entre el Discurso sobre los origenes y fundamentos de la desigualdad entre los hombres y El contrato social
}

\author{
Juan Camilo Gallo-Gómez \\ Universidad de Medellín \\ jcgallo@udem.edu.co \\ https://orcid.org/0000-0003-2920-2194
}

Resumen: Este artículo busca presentar el legado teórico-político de Rousseau a través de la interpretación de su Discurso sobre los origenes y fundamentos de la desigualdad entre los hombres y el Contrato social, entendidos como una unidad argumentativa y metodológica en la obra del ginebrino. Para esto se expondrán en un primer momento las consideraciones normativas y antropológicas que marcan el problema político de Rousseau. A partir de alli se presentarán las ideas principales de su noción de democracia, para finalmente concluir que el legado democrático de Rousseau resulta particularmente útil como elemento crítico en el contexto de la hegemonía de las democracias representativas liberales actuales. Palabras clave: Rousseau; democracia; igualdad; libertad; deliberación

\begin{abstract}
Rousseau's Philosophical-political Program: the Theoretical Link between the Discourse on the Origins and Foundations of the Inequality Among Mankind and the Social Contract'. This paper presents Rousseau's political and theoretical legacy through the interpretation of his Discourse on the Origins and Foundations of the Inequality Among Mankind and the Social Contract, understanding both as a sole argumentative and methodological unit in his work. In order to achieve this objective, the paper explores first the normative and anthropological considerations that mark the problem of Rousseau's political theory. From there, the main ideas of his notion of democracy will be presented. Finally, the paper concludes that Rousseau's democratic legacy is particularly useful as a form of critique in the hegemonic context of the current liberal and representative democracies.
\end{abstract}

Keywords: Rousseau; democracy; equality; freedom; deliberation 


\section{Introducción}

Jean Jacques Rousseau es uno de los pensadores más importantes de la historia de la filosofia política. Se ha ganado este reconocimiento a través de su fecundidad literaria y de la relevancia de sus ideas para la reflexión filosófico-política en torno a los principios justos de la organización social ${ }^{1}$. Su importancia se ve reflejada, además, en la multiplicidad de críticas que se le han formulado desde diferentes puntos de vista. Tanto autores conservadores como Edmund Burke, Joseph de Maistre o Leo Strauss, y pensadores liberales como Benjamin Constant, Bertrand Russell o Isaiah Berlin, han hecho a la obra del ginebrino sendas críticas que, justificadas o no, han generado una opinión común sobre Rousseau como un enemigo de la propiedad privada, de las libertades individuales y como un defensor del totalitarismo de la mayoría ${ }^{2}$ No en vano se le culpa del terror jacobino durante la Revolución Francesa ${ }^{3}$.

El objetivo principal de este artículo consiste en defender precisamente la herencia democrática de Rousseau a través de la coherencia argumentativa entre el Discurso sobre los origenes y fundamentos de la desigualdad entre los hombres y el Contrato social, de cara a la representación generalizada que se ha hecho del ginebrino, principalmente a partir de Isaiah Berlin. Estas críticas, comenzando con Voltaire, han girado alrededor de los siguientes aspectos que aparentemente se deducen de las ideas del Contrato social: 1) La primacía de la comunidad sobre el individuo, lo que implica la negación de las libertades individuales y la defensa de una suerte de tiranía de la mayoría; 2) la supremacía de las libertades positivas -como la participación politica-sobre las libertades negativas propias del liberalismo de la modernidad, afirmación que conlleva el hecho de la supuesta preferencia de Rousseau por las prácticas políticas de la antigua Grecia, lo que convierte al ginebrino en un nostálgico anti-moderno, simpatizante de la autoridad absoluta de la ciudad-estado y de la rendición del individuo a la comunidad ${ }^{4}$; 3) la idea de que la voluntad general niega los intereses fundamentales de las personas al verse subsumidos por el macro sujeto, el pueblo, que detenta dicha voluntad. La lista podría ampliarse; sin embargo, estas tres ideas presentadas pueden dar una perspectiva general sobre

$4 \quad$ Ibid., pp. 76-77. 
la opinión común que se ha tenido de la obra del ginebrino: como un enemigo de las libertades democráticas propias de la modernidad politica, siendo su legado filosófico-político el totalitarismo y terror de Robespierre, Hitler y Mussolini ${ }^{5}$.

Con el objetivo de presentar una perspectiva alternativa frente a estas críticas, en este artículo se plantea la importancia filosófica y política de Rousseau para los debates contemporáneos sobre la democracia, al enfocarse precisamente en la relación de reciprocidad que existe entre libertad e igualdad como los intereses generales de los ciudadanos, que configuran la idea de la voluntad general como el marco político para la cooperación social y el consenso $^{6}$. Para poder justificar esta interpretación, se comenzará presentando el problema central del Contrato socialy su estrecha relación metodológica con las críticas a la sociedad moderna presentadas por Rousseau en el Discurso sobre los origenes y fundamentos de la desigualdad entre los hombres (1); tras este planteamiento, se expondrá la concepción de democracia del ginebrino a partir de las ideas de la libertad, la igualdad, el pluralismo y la participación ciudadana en la toma de decisiones (2); por último, se presentarán las conclusiones que buscan señalar el valor crítico actual de la teoría republicana de la democracia del ginebrino con respecto a la hegemonía de los modelos liberales representativos (3).

\section{El problema central del Contrato Social}

\subsection{El punto de partida: el discurso sobre la desigualdad entre los hombres}

En 1755 aparece el Discurso sobre los origenes y fundamentos de la desigualdad entre los hombres, con el cual Rousseau esperaba responder a la cuestión propuesta por la Academia de Dijon sobre "cuál es el origen de la desigualdad entre los hombres y si está autorizada por la ley natural". Desde las primeras líneas de su ensayo, Rousseau presenta la idea de que sólo hay

\footnotetext{
5 Berlin es bastante injusto con varios filósofos, particularmente con Hegel y Rousseau, a quienes acusa de ser racionalistas dogmáticos, simplistas, metafísicos y enemigos de la libertad individual. Esto puede deberse a la lectura de la versión inglesa de Ernst Vaughan de las obras de Rousseau, quien sostenía que el ginebrino era un colectivista radical. Además, Berlin tomó como objetivo filosófico-político, la defensa de la llamada libertad negativa, observando detrás de las libertades positivas un supuesto despotismo democrático, en el contexto de la Guerra Fría. Sin embargo, esta contextualización no exime a Berlin del pobre tratamiento que le da a Rousseau y otros pensadores. Cf. Berlin, I., La traición de la libertad. Seis enemigos de la libertad humana, México: Fondo de Cultura Económica, 2004, pp. 66-69; Arango, I.D., Críticos y lectores de Rousseau. Medellin: Editorial Universidad de Antioquia, 2006, pp. 98-99.

6 Cf. Arango, I.D., Críticos y lectores de Rousseau; De Dijn, A., "Rousseau and Republicanism", en: Political Theory, v. XLVI, 1 (2018), pp. 59-80.
} 
un tipo de desigualdad prescrito por la ley natural, y esta es la desigualdad en las dotaciones innatas, en los talentos o fortalezas que cada individuo posee. Sin embargo, hay una segunda clase de desigualdad, que es llamada moral o política, y se refiere a las diferencias de poder, riqueza y prerrogativas sociales de las cuales disfrutan ciertos individuos sobre los demás. Hay dos problemas aquí: primero, al no ser este tipo de desigualdad propio de la naturaleza, debe ser autorizado a través de convenciones humanas; segundo, parece ser que en la sociedad civil las desigualdades naturales no se corresponden con las desigualdades morales o políticas, en otras palabras, aquellos que poseen el poder, la riqueza y una serie de beneficios sociales sobre los demás no parecen ser siempre los más talentosos o virtuosos. Teniendo esto en cuenta, nuestro autor se pregunta lo siguiente: ¿cómo ha podido una mayoría aceptar su sometimiento al poder de unos pocos?

Para responder esta pregunta, es necesario partir de la concepción normativa de la persona en el estado de naturaleza que plantea Rousseau. En dicho estado, los seres humanos son seres primitivos, sin uso de razón y sin pasiones ni vicios que orienten sus acciones, haciendo una clara critica al concepto hobbesiano de estado de naturaleza ${ }^{7}$. Sólo hay dos principios naturales que guían los actos de los seres humanos en el estado de naturaleza, de los cuales "...uno nos interesa vivamente para bienestar nuestro y para la conservación de nosotros mismos, y el otro nos inspira una repugnancia natural a ver perecer o sufrir a cualquier ser sensible, especialmente nuestros semejantes". Estos dos principios son el amor de sí mismo (amour de soi-même) y la piedad (pitié), los cuales, aunados a la perfectibilidad (perfectibilité) como la capacidad humana de transformar sus propias condiciones subjetivas y sus contextos intersubjetivos de acción, forman el conjunto normativo del concepto de persona en la obra del ginebrino ${ }^{9}$. La condición de perfectibilidad humana señalada por Rousseau ha sido generalmente asumida como la posibilidad de mejorar constantemente para alcanzar el potencial de los seres humanos. Sin embargo, aquí se entiende perfectibilidad como la posibilidad siempre presente de transformación humana, sin objetivos determinados, es decir, como la capa-

Cf. Thomä, D., Puer Robustus. Una filosofia del perturbador, Barcelona: Herder, 2018, p. 71.

Rousseau, J.J., Discurso sobre el origen y los fundamentos de la desigualdad entre los hombres. Discurso sobre las ciencias y las artes. Del Contrato social, Madrid: Alianza Editorial, 2008, pp. 224-225.

9 Ibid., p. 245. 
cidad humana de actuar libremente y su capacidad de adaptación, que puede verse tanto en sentido progresivo como regresivo ${ }^{10}$.

Este estado natural está caracterizado, además, por la independencia de cada una de las personas frente a las demás, dado que cada una solo se guía por sus necesidades básicas y el instinto, los lazos sociales no existen y son superfluos para el desarrollo de las vidas de las personas. En esta medida, la situación primigenia de los seres humanos no es un estado de guerra de todos contra todos, sino una situación pacífica propiciada por el aislamiento de los individuos que lo conforman ${ }^{11}$. Esto implica que las condiciones naturales de los seres humanos son dos: la libertad en el sentido de una independencia frente a cualquier voluntad diferente a la propia, y la igualdad, en el sentido de que todas las personas poseen los mismos principios naturales: amor de sí mismo, piedad y la capacidad de la perfectibilidad.

No obstante, esta representación del estado de naturaleza no implica una reminiscencia ingenua de "todo tiempo pasado fue mejor"; por el contrario, la descripción antropológica que realiza Rousseau en su Discurso sobre la desigualdad o Segundo discurso, se entrelaza estrechamente con el problema político de la legitimación de la autoridad que abordará posteriormente, en 1762, en su Contrato social ${ }^{12}$. En este sentido, la condición natural de los seres humanos determina las consideraciones posteriores sobre los principios justos de organización social. De aquí se sigue que el problema central del pacto social sea precisamente el establecimiento de los términos justos para la convivencia y cooperación social, y no una vuelta a aquel estado primigenio, dado que la convivencia y cooperación aparecen como la respuesta a los obstáculos que la naturaleza les impone a los individuos en el estado natural, tal como se plantea en el libro primero del Contrato social: "Parto de considerar a los hombres llegados a un punto en el que los obstáculos que dañan a su conservación en el estado de naturaleza logran superar, mediante su resistencia, la fuerza que cada individuo puede emplear para mantenerse en ese estado. Desde ese momento tal estado originario no puede subsistir y el género humano perecería si no cambiase de manera de ser"13.

${ }^{10} C f$. Thomas, P., "Among Prelates and Primates. From Darwin to Rousseau", en: Political Theory, v. XXXVII, 34 (2009), p. 457.

${ }^{11}$ Cf. Kersting, W., Jean Jacques Rousseaus "Gesellschafts Vertrag", Darmstad: Wissenschaftliche Buchgesellschaft, 2002, p. 25.

${ }^{12}$ Ibid., pp. 17-18.

${ }^{13}$ Rousseau, J.J. Discurso sobre el origen y los fundamentos de la desigualdad entre los hombres. Discurso sobre las ciencias y las artes. Del Contrato social, pp. 37-38. 
Así, la cooperación y la convivencia se vuelven indispensables para la supervivencia de la humanidad. La sociedad surge entonces como una forma para enfrentar las necesidades individuales a través del trabajo cooperativo. Esto lleva a nuestro autor a reconocer tres momentos en el desarrollo social de la humanidad que siguen a la situación pre-social del estado de naturaleza: 1) Sociedades pequeñas de cazadores y recolectores que encuentran más eficiente el trabajo en equipo, aunque sin lazos de sociabilidad aún; 2) sociedades tribales centradas en torno a agrupaciones de familias donde la propiedad es generalizada y está repartida entre sus miembros, donde aparecen los primeros rasgos de sociabilidad, como el amor, el honor y los deseos de reconocimiento; y 3) la sociedad moderna que encuentra su origen en las convenciones y generaliza los males sociales a través de la introducción de las desigualdades morales y políticas con la acumulación de la propiedad privada y la dominación de los más débiles por parte de los más fuertes. Este desarrollo histórico de la humanidad, que Rousseau deja como un relato entre la ficción y la realidad ${ }^{14}$, tiene como objetivo mostrar el proceso de desnaturalización de las personas mediante el avance técnico-social que conlleva la aparición de nuevos desafios políticos y sociales. Así, las necesidades crecientes de cooperación social generan estructuras organizacionales cada vez más complejas que exigen nuevas formas de justificación del ejercicio del poder político.

Dado que Rousseau busca explicar las causas de las condiciones de desigualdad politica y social, dirige todas sus críticas a la sociedad moderna, cuyo fundamento se encuentra en la propiedad privada. La segunda parte del Segundo discurso comienza precisamente haciendo referencia a la introducción de la propiedad privada como el factor que determina la salida del estado social pre-moderno anterior, en el cual existía propiedad común sobre la tierra y algunos vínculos sociales básicos, y el comienzo de la desigualdad y la opresión políticas: "El primero al que, tras haber cercado un terreno, se le ocurrió decir 'esto es mío' y encontró personas lo bastante simples para creerle, fue el verdadero fundador de la sociedad civil" ${ }^{15}$. Con esta idea, generalmente tomada como un desprecio al derecho a la propiedad privada, Rousseau se convierte en el primer crítico de la modernidad, puesto que señala dos puntos que siguen estando vigentes actualmente. En primer lugar, toda la sociedad moderna, y

\footnotetext{
${ }^{14}$ Cf. Thomä, Puer Robustus. Una filosofia del perturbador, p. 73; Garrard, G., "Rousseau: Happiness and Human Nature”, en: Political Studies, v. LXII (2014), p. 70.

${ }^{15}$ Rousseau, J.J., Discurso sobre el origen y los fundamentos de la desigualdad entre los hombres. Discurso sobre las ciencias y las artes. Del Contrato social, p. 276.
} 
con ella todo su proyecto de emancipación política, está fundamentada sobre la propiedad privada ${ }^{16}$. Es el reconocimiento institucionalizado en el Estado absoluto moderno de derechos individuales de propiedad sobre la tierra y los medios de producción lo que en realidad marca la transición de la Edad Media a la modernidad propiamente hablando. No son ni la centralidad de la razón ni "las luces" de la nueva ciencia los factores determinantes, es el cambio del sistema de producción lo que genera la transformación social y los criterios de justificación del ejercicio del poder ${ }^{17}$.

En segundo lugar, Rousseau critica las presuposiciones liberales de la naturalidad del derecho a la propiedad privada. John Locke ya había señalado que: "[Dios] Ha dado el mundo para que el hombre trabajador y racional lo use; y es el trabajo lo que le da derecho a la propiedad, y no los delirios y la avaricia de los revoltosos y los pendencieros"18. Para Rousseau, por el contrario, la propiedad privada no puede ser producto de la ley natural, aunque tampoco es contraria a esta. La propiedad privada es producto de la capacidad humana de transformarse a sí mismo y a su entorno y en sí misma no es mala, aunque, al ser un producto humano, necesita ser justificada y legalizada por sistemas legislativos ${ }^{19}$. El problema para nuestro autor consiste en que un sistema económico basado en la acumulación de la propiedad sobre los medios de producción necesariamente genera conflictos sociales que deben ser enfrentados mediante la justificación de la desigualdad socio-económica ${ }^{20}$.

Rousseau atribuye, entonces, el surgimiento de la sociedad moderna a la creación del sistema de producción basado en la propiedad privada. A partir de este acto originario se siguieron otros hechos que fomentaron la desigualdad social: la acumulación de capital, la división del trabajo, la competencia entre las dotaciones innatas de los individuos y la transferencia de la propiedad acumulada de padres a hijos a través de la herencia. Estos factores crearon la primera de las dicotomias sociales: la diferencia entre los ricos y los pobres, los primeros rebosando de beneficios, los segundos careciendo de lo necesario ${ }^{21}$.

${ }^{16}$ Cf. Hardt, M. y A. Negri, Commonwealth, Cambridge/Londres: Harvard University Press, 2009, p. 9.

17 Cf. Anderson, P., El Estado absolutista, Madrid: Siglo XXI, 2016, p. 27.

${ }^{18}$ Locke, J., Segundo tratado sobre el gobierno civil, Madrid: Alianza Editorial, 2010, p. 61.

${ }^{19}$ Cf. Pierson, C., "Rousseau and the Paradoxes of Property", en: European Journal of Political Theory, v. XII, 4 (2013), p. 411.

${ }^{20}$ Cf. Kersting, W., Jean Jacques Rousseaus "Gesellschafts Vertrag", p. 25.

21 Cf. Pierson, C., "Rousseau and the Paradoxes of Property", p. 414. 
A través de la acumulación del capital, los ricos tuvieron el poder de decidir sobre los pobres, asociándose así los monopolios económicos con el monopolio del poder, surgiendo la segunda forma de desigualdad social: los poderosos y los débiles. Sin embargo, los poderosos se vieron en la necesidad de dotar de legitimidad su poder, dado que "el más fuerte nunca es bastante fuerte para ser siempre el amo si no transforma su fuerza en derecho y la obediencia en poder" 22. Engañando a los débiles, los ricos y poderosos les hicieron creer que les servian a ellos cuando en realidad los explotaban. Este proceso se conoce como el contrato falso o fraudulento, y se puede considerar un primer diagnóstico del papel de la ideología, entendida como falsa conciencia ${ }^{23}$, en la justificación, reproducción y legitimación de las relaciones de dominación. Kersting ve en esta formulación rousseauniana de la transición a la Modernidad, el primer esfuerzo moderno de crítica a las estructuras ideológicas de dominación: " $\mathrm{El}$ contrato entre ricos y pobres profundiza las desigualdades y el orden injusto del estado de naturaleza social a través de la legalización formal. Es un contrato fraudulento que los ricos utilizan como un instrumento sofisticado para sus intereses..."24.

En este momento, el ser humano que, por naturaleza, era libre e igual se ve sometido al dominio de unos cuantos, y degradado así de su condición humana. Aquí surge la peor desigualdad de todas: la existente entre los amos y los esclavos, que conlleva consigo todos los males sociales, contrarios a la naturaleza humana. Tal como Rousseau dice: "Se desprende además que la desigualdad moral, solamente autorizada por el derecho positivo, es contraria al derecho natural, siempre que no concurra en igual proporción con la desigualdad física (en el sentido de virtudes y talentos propios de cada individuo); distinción que determina suficientemente lo que debe pensarse de la clase de desigualdad que reina entre todos los pueblos civilizados, puesto que va manifiestamente contra la ley de la naturaleza, de cualquier forma que

${ }^{22}$ Rousseau, J.J., Discurso sobre el origen y los fundamentos de la desigualdad entre los hombres. Discurso sobre las ciencias y las artes. Del Contrato social, p. 29.

${ }^{23}$ Cabe aclarar que no se está equiparando a Rousseau con Marx. Esta afirmación busca establecer un vínculo hermenéutico entre ambos pensadores, sin afirmar que el ginebrino es una anticipación del pensamiento marxista. Cf. Althusser, L., Lessons on Rousseau, Londres: Verso, 2019.

${ }^{24}$ Der Vertrag zwischen den Reichen und Armen vertieft die Ungleichheits- und Ungerechtigkeitsordnung des gesellschaftlichen Naturzustandes durch formale Verrechtlichung. Es ist ein Täuschungs- und Betrugsvertrag, den die Reichen als raffiniertes Instrument ihrer Interessen handhaben (Kersting, W., Jean Jacques Rousseaus "Gesellschafts Vertrag”, p. 27). Traducción al español mía. 
se la defina, el que un niño mande a un anciano, el que un imbécil guie a un hombre sabio y el que un puñado de gentes rebose de superfluidades mientras la multitud hambrienta carece de lo necesario"25.

De lo expuesto hasta aquí pueden desprenderse dos afirmaciones relacionadas entre sí: el hombre es bueno por naturaleza y la sociedad lo corrompe, y las desigualdades sociales y la opresión no provienen de las condiciones naturales, es decir, atentan contra la humanidad, son producto de relaciones de poder establecidas dentro la sociedad moderna para la protección de los intereses de grupos particulares y se han legitimado a través de principios de justificación engañosos o fraudulentos. Es necesario precisar el sentido de estas dos afirmaciones, pues se relacionan con el problema central del Contrato social. En primer lugar, la afirmación de que el hombre es bueno por naturaleza y la sociedad lo corrompe puede entenderse como la forma en que las instituciones sociales afectan las posibilidades de desarrollo de las capacidades naturales de las personas, determinadas por los principios naturales ya mencionados del amor de sí mismo y la piedad aunadas a la perfectibilidad. El amor de sí mismo y la piedad se ven reemplazados por el amor propio (amour propre) como una forma artificial de estima personal que depende de las opiniones de los demás, despertando así la vanagloria y los conflictos interpersonales ${ }^{26}$. Las instituciones politicas injustas que oprimen a los ciudadanos y producen desigualdades socioeconómicas, solo pueden generar conflictos sociales entre los intereses particulares contrapuestos, de donde se sigue la necesidad de legitimar, mediante un pacto fraudulento, un engaño, las estructuras de dominación como un intento por salvaguardar los beneficios y ventajas de los más poderosos.

En segundo lugar, si la opresión y las desigualdades injustificadas de la sociedad moderna atentan contra la humanidad, resulta necesario entonces superar esta condición a través del establecimiento de una sociedad que se rija por principios políticos que puedan ser considerados como justos por todos los miembros de la comunidad. Este es precisamente el problema central del programa filosófico de Rousseau ${ }^{27}$. Este programa ha sido interpretado de forma inadecuada, principalmente a partir de Berlin, quien sostiene que la idea de contrato en la obra de Rousseau autoriza la tiranía de la mayoría y confunde

\footnotetext{
${ }^{25}$ Rousseau, J.J., Discurso sobre el origen y los fundamentos de la desigualdad entre los hombres. Discurso sobre las ciencias y las artes. Del Contrato social, p. 316.

${ }^{26}$ Cf. Garrard, G., "Rousseau: Happiness and Human Nature", pp. 73-74.

${ }^{27}$ Cf. Rubio, J., "Rousseau y Kant: una relación proteica", en: Revista de Estudios Politicos, 133 (2006), p. 22.
} 
libertad con autoridad. Tras acusar a Rousseau de ser un maniático, Berlin dice: "Hay una libertad que es idéntica a la autoridad, y es posible tener una libertad personal que es la misma como un completo dominio por parte de la autoridad. Cuánto más libre se es, más autoridad se tiene y también más se obedece; cuanta más libertad más dominio"28.

El problema fundamental en la interpretación liberal de Berlin de Rousseau radica en que este no va más allá de la idea de libertad entendida en términos puramente negativos, de ausencia de intervención de la autoridad en los asuntos privados ${ }^{29}$. Esto le impide entender las nociones republicanas de libertad como autonomía que presenta Rousseau y que van a ser retomadas posteriormente por Kant ${ }^{30}$. En este sentido, el programa filosófico-político de Rousseau va más allá de la noción de libertad puramente negativa del liberalismo clásico al considerar que los principios de organización social no pueden estar simplemente fundamentados en la agregación de intereses particulares, debido a los problemas de desigualdad y opresión que generan las estructuras de poder en la sociedad ${ }^{31}$.

Este programa puede entenderse en tres momentos. El primero es la definición normativa de la persona a través del argumento de la naturaleza humana en un estado pre-político y su evolución a través de procesos de adaptación al entorno cambiante ${ }^{32}$. Esta naturaleza está definida por la libertad e igualdad de todos los seres humanos. Un segundo momento que se establece a través de la negación de la condición natural del hombre mediante el establecimiento del contrato social fraudulento entre ricos y pobres, que es definido de la siguiente manera: "Yo hago contigo un convenio, enteramente en perjuicio tuyo y enteramente en beneficio mío, que yo observaré mientras me plazca, y que tú observarás mientras me plazca"33. Este segundo momento es el de la desnaturalización de las personas a través de la opresión, la tiranía y la desigualdad social. Estas dos fases del programa normativo rousseauniano son abordadas en el Segundo discurso. Queda pues el tercer momento, el de la re-naturalización de las personas a través de la creación de un pacto social

\footnotetext{
28 Berlin, I., La traición de la libertad. Seis enemigos de la libertad humana, p. 61.

${ }^{29}$ Cf. Berlin, I., Dos conceptos de libertad. El fin justifica los medios. Mi trayectoria intelectual, p. 82 .

${ }^{30} C f$. Rubio, J., "Rousseau y Kant: una relación proteica”, p. 11.

31 Cf. De Dijn, A., "Rousseau and Republicanism”, p. 61.

32 Cf. Thomas, P., "Among Prelates and Primates. From Darwin to Rousseau", p. 468.

33 Rousseau, J.J., Discurso sobre el origen y los fundamentos de la desigualdad entre los hombres. Discurso sobre las ciencias y las artes. Del Contrato social, p. 36.
} 
justo, y este es el problema central de la filosofia politica del ginebrino en su Contrato social.

1.2. El contrato social como enfoque normativo frente al despotismo y la desigualdad.

Frente a la necesidad de la cooperación social para la supervivencia de la especie humana, resulta indispensable para Rousseau, sentar las bases justas para la convivencia social bajo instituciones politicas. Estas bases justas son las que les permitirán a los seres humanos recobrar su libertad e igualdad esenciales a su naturaleza, perdidas a través de la opresión y la iniquidad social que se ha legitimado mediante el contrato fraudulento. Rousseau plantea el problema de la siguiente manera: "Esta dificultad aplicada a mi tema puede enunciarse en los siguientes términos: 'Encontrar una forma de asociación que defienda y proteja de toda la fuerza común la persona y los bienes de cada asociado, y por la cual, uniéndose cada uno a todos, no obedezca, sin embargo, más que a sí mismo y quede tan libre como antes'. Tal es el problema fundamental al que da solución el contrato social"34.

Este problema puede entenderse como la forma mediante la cual podemos unirnos con los demás sin sacrificar nuestra libertad esencial y, de esta manera, poder promover nuestros intereses fundamentales a través del marco social que se origina con el pacto fundante. La respuesta a este problema se encuentra en la definición del contenido normativo del contrato que todos los participantes acuerdan. El consenso sobre las bases justas de la organización social se consolida entonces como la única forma legítima para el establecimiento de los términos de la cooperación social y como el mecanismo de legitimación de la obligación política. Este contenido es definido por el ginebrino en los siguientes términos: "Cada uno de nosotros pone en común su persona y todo su poder bajo la suprema dirección de la voluntad general, y nosotros recibimos corporativamente (en corps) a cada miembro como parte indivisible del todo" 35 .

En este punto aparece la idea de la voluntad general, concepto central de la filosofia política rousseauniana y el foco del mayor número de críticas hechas a su obra. ¿Qué es la voluntad general? Este concepto debe entenderse como el principio normativo que establece el marco básico para la organización social y politica de la comunidad a través del principio de reciprocidad ${ }^{36}$.

\footnotetext{
34 Ibid., p. 39.

35 Ibid.

${ }^{36}$ Cf. Kersting, W., Jean Jacques Rousseaus "Gesellschafts Vertrag”, p. 27; Schwartzberg, M., "Rousseau on Fundamental Law", en: Political Studies, v. LI (2003), p. 388.
} 
En este sentido, al unirse todos los individuos en un cuerpo colectivo a través del pacto social, buscan la promoción de sus intereses fundamentales como individuos que forman parte de una colectividad. Sin embargo, además de los intereses fundamentales, las personas también poseen intereses particulares que pueden chocar entre sí. Por esta razón, es necesario establecer un punto de consenso para poder dirigir esos diferentes intereses hacia el mismo fin, el cual es la conservación de cada uno de los miembros del cuerpo colectivo a través de la cooperación social, sin privar de la libertad ni la igualdad moral y política a ninguna de las partes contratantes.

De esta manera, la voluntad general representa lo que cada uno de los individuos quiere como parte indispensable del todo, es decir, la voluntad general representa los intereses generales de todos los miembros de la sociedad o, en otras palabras, lo que es bueno para todos siguiendo un criterio de reciprocidad en la justicia. Este es un criterio público que se refiere a las decisiones que afectan o pueden afectar a todos como ciudadanos del cuerpo político, miembros del pueblo soberano y como partes del mismo, es decir, aquellas decisiones que afectan a las personas tanto como pueblo y como individuos, dado que las personas son interdependientes -necesitan de la cooperación con los otros-, pero poseen al mismo tiempo el interés natural de auto-preservación; individuo y sociedad no pueden separarse ${ }^{37}$. De aquí se sigue que el todo no puede sacrificar a ninguna de sus partes $^{38}$.

Estos intereses generales representan la humanidad de las personas, es decir, las condiciones naturales que hacen al ser humano ser lo que es. Estas condiciones son la libertad, entendida como no estar sometido a ninguna autoridad distinta a la propia y la igualdad moral de cada uno de los miembros del pueblo. Tal como el ginebrino sostiene: "Si se indaga en que consiste el mayor bien de todos, que debe ser el fin de todo sistema de legislación, se encontrará que se reduce a dos objetos principales, la libertad y la igualdad. La libertad, porque toda dependencia particular es otro tanto de fuerza que se quita al cuerpo del Estado; la igualdad, porque la libertad no puede subsistir sin ella"39.

Solo en la medida en que las personas acuerdan sumar su persona y sus bienes a la consecución de los intereses generales (libertad e igualdad) de

\footnotetext{
37 Cf. Cohen, J., Rousseau. A Free Community of Equals, Oxford: Oxford University Press, 2010, p. 25.

${ }^{38}$ Cf. Rousseau, J.J., Discurso sobre el origen y los fundamentos de la desigualdad entre los hombres. Discurso sobre las ciencias y las artes. Del Contrato social, p. 41.

39 Ibid., p. 71.
} 
cada una de las partes contratantes, es posible originar el cuerpo colectivo o la sociedad entendida como un sistema de cooperación equitativa, y en esta medida, surge el pueblo soberano, compuesto por cada uno de los ciudadanos reunidos en asamblea, como el detentador de la suprema autoridad en la sociedad. Esta idea del soberano en Rousseau está estrechamente unida a la idea de la voluntad general, pues cuando las personas acuerdan trabajar en conjunto para el bien común pueden concebirse a sí mismos como miembros de una comunidad. En este punto es necesario hacer dos aclaraciones: primero, la voluntad general no es externa a los mismos individuos, es decir, la voluntad general no la detenta nadie en particular, ni siquiera la comunidad entendida en conjunto como un macro sujeto que subordina a las personas a la consecución de los fines colectivos. En este sentido, la voluntad general está conformada por los intereses fundamentales de cada una de las partes, intereses que corresponden con la definición normativa de las personas como libres e iguales ${ }^{40}$. De este punto se sigue que la voluntad general no aspira a regular absolutamente todos los aspectos de la vida de las personas, sino sólo aquellos que puedan afectar la consecución del bien común, es decir, la voluntad general se aplica a los asuntos públicos, no a la esfera privada de los individuos, a menos que los intereses privados de algunos individuos afecten el interés general de libertad e igualdad, por ejemplo en el caso de que una minoría (los ricos) quieran justificar una forma injusta de cooperación colectiva. Puede decirse, además, que la voluntad general no niega los intereses particulares de los individuos, por el contrario, la idea de la voluntad general busca promover la consecución de dichos intereses a través de un marco social consensuado que trate a cada miembro de la sociedad como una parte indispensable para la vida en común ${ }^{41}$.

La segunda aclaración es con respecto al soberano. Este está conformado por cada uno de los miembros de la sociedad en tanto que son ciudadanos. Por esta razón, el soberano no representa un poder diferente al que cada individuo posee como participante en las deliberaciones propias de las asambleas. El soberano no es, pues, un poder diferente al de los miembros de la sociedad. Rousseau dice: "Tan pronto como esta multitud se encuentra así reunida en un cuerpo, no se puede ofender a uno de los miembros sin atacar el cuerpo... Ahora bien, al no estar formado el soberano más que por los particulares que lo componen, no tiene ni puede tener interés contrario al suyo; por consiguiente, el

${ }^{40}$ Cf. Kersting, W., Jean Jacques "Rousseaus Gesellschafts Vertrag", p. 82.

${ }^{41}$ Cf. Cohen, J., Rousseau. A Free Community of Equals, pp. 24-25.

ARETÉ Revista de Filosofia, v. XXXIII, 1, 2021 / e-ISSN 2223-3741 
poder soberano no tiene ninguna necesidad de garantía respecto a los súbditos, porque es imposible que el cuerpo quiera perjudicar a todos sus miembros, y luego veremos que no puede afectar a ninguno en particular" 42 .

A través del pacto social que implica el consenso sobre los intereses generales que conforman la voluntad general, surge el soberano, conformado por cada uno de los miembros de la sociedad en tanto libres e iguales. Por medio del soberano se expresa la voluntad general en la forma de las leyes politicas fundamentales que conciernen a las cuestiones de la justicia básica y a las esencias constitucionales ${ }^{43}$. El poder soberano, entendido entonces como el poder del pueblo reunido en asamblea como cuerpo colectivo, es el poder constituyente y, en este sentido, el pueblo reunido en asamblea establece los principios políticos básicos de la sociedad ${ }^{44}$, como la forma de la organización institucional y la adscripción de derechos fundamentales a cada uno de los miembros del cuerpo politico. A través de la voluntad general se genera también una relación de sujeción a la ley como el medio necesario para el aseguramiento de las libertades básicas de todos. Las personas poseen un doble carácter político: como ciudadanos miembros del soberano y poseedores por consiguiente del poder constituyente, y como súbditos, miembros del estado y sujetos a las leyes que ellos mismos se dan. Frente a este asunto, cabe preguntarse: ¿Cómo podemos ser libres y al mismo tiempo estar sujetados a las leyes? ¿Cuál es la relación entre la libertad positiva y la negativa en el Contrato social? ¿Cómo se relacionan estas ideas con el concepto de democracia en la obra de Rousseau?

\section{Libertad, igualdad, pluralismo y deliberación}

Hasta el momento se ha sostenido que el objetivo del contrato social es permitir que las personas conserven su esencia humana, constituida por la

42 Rousseau, J.J., Discurso sobre el origen y los fundamentos de la desigualdad entre los hombres. Discurso sobre las ciencias y las artes. Del Contrato social, p. 41.

43 Cf. Schwartzberg, M., "Rousseau on Fundamental Law", p. 392.

44 No en vano el subtítulo del Contrato social es precisamente Principios del derecho politico.

108 Este subtítulo es fundamental para entender el objetivo de Rousseau con su obra. Estos principios, siguiendo a Derathé pueden resumirse así: 1) Nadie tiene un poder natural sobre sus semejantes, ningún poder es legítimo sino cuenta con el consentimiento del pueblo; 2) el poder soberano reside en el pueblo y solamente en él, no puede ser entregado ni alienado; 3) el gobierno es diferente del soberano, sus funciones públicas se limitan a la administración autorizada por el pueblo. El gobierno no es más que un ejercicio administrativo subordinado al poder soberano. Así, de acuerdo con Derathé, estos principios representan la base de las democracias constitucionales contemporáneas, más que representar algún tipo de democracia de carácter populista. $C f$. Derathé, R., Rousseau et la Science Politique de son temps, Paris: Libraire philosophique J. Vrain, 1995, pp. 49-50. 
libertad y la igualdad. En lo que se refiere a la libertad, el ginebrino sostiene que "Renunciar a su libertad es renunciar a su cualidad de hombre, a los derechos de la humanidad, incluso a sus deberes. No hay compensación posible para quien renuncia a todo. Semejante renuncia es incompatible con la naturaleza del hombre, y es privar de toda moralidad a sus acciones el privar a su voluntad de toda libertad. En fin, es una convención vana y contradictoria estipular por un lado una autoridad absoluta, y por otro una obediencia sin límites"45.

Por esta razón, el problema teórico rousseauniano hace un énfasis en la posibilidad de conservar la libertad en un contexto de interdependencia social mutuamente ventajosa. De otra manera, el contrato fundante atentaría contra la naturaleza humana y carecería de toda legitimidad ${ }^{46}$.

Rousseau distingue tres formas de libertad: la natural, la civil y la moral. La primera de estas formas de libertad, hace referencia a la independencia de cualquier otra voluntad externa al individuo. En el estado de naturaleza, los individuos se guian únicamente por sus necesidades e instinto, teniendo así un derecho ilimitado a lo que desean y están en capacidad de conseguir. Esta libertad se pierde con la creación de la sociedad a través del pacto social; sin embargo, los individuos ganan otras dos formas de libertad, más apropiadas para la vida en común: la libertad civil, que implica la capacidad de acción limitada exclusivamente por la voluntad general, es decir, limitada por el reconocimiento recíproco de las libertades de los demás, y la libertad moral, que implica guiarse exclusivamente por las leyes que uno mismo se ha prescrito, es decir, la idea de autonomía que tanta influencia va a tener posteriormente en Kant ${ }^{47}$. Con la sociedad civil y el contrato justo no solo logramos superar los obstáculos que nos impone la naturaleza para la conservación de la especie, sino que además conseguimos dotar de moralidad a nuestras acciones: "Este paso del estado de naturaleza al estado civil produce en el hombre un cambio muy notable, sustituyendo en su conducta el instinto por la justicia, y dando a sus acciones la moralidad que les faltaba antes. Solo entonces, cuando la voz del deber sucede al impulso físico y el derecho al apetito, el hombre que

\footnotetext{
${ }^{45}$ Rousseau, J.J., Discurso sobre el origen y los fundamentos de la desigualdad entre los hombres. Discurso sobre las ciencias y las artes. Del Contrato social, p. 32.

${ }^{46}$ Cf. Cohen, J., Rousseau. A Free Community of Equals, p. 30; Kersting, W., Jean Jacques Rousseaus "Gesellschafts Vertrag", p. 79.

${ }^{47} C f$. Rubio, J., "Rousseau y Kant: una relación proteica", p. 45.
} 
hasta entonces no había mirado más que a sí mismo, se ve forzado a obrar por otros principios y a consultar su razón antes de escuchar sus inclinaciones" 48 .

La libertad civil y moral se encuentran en una relación de reciprocidad: solamente puedo ser libre cuando me guío por las leyes y estas han sido aprobadas por mí como miembro del soberano. En este punto se conjugan las dos libertades: la de los antiguos y la de los modernos, utilizando la misma expresión de Constant ${ }^{49}$. La libertad de los antiguos se expresa a través de la autolegislación que realizan los individuos como detentadores de la soberanía y del poder constituyente. Esto quiere decir que sólo son legítimas aquellas normas o principios políticos que concuerden con el interés general, que ha sido establecido por las personas a través del consenso en torno al contenido normativo del contrato originario. La libertad de los modernos se encuentra en el respeto por la esfera privada de las personas, limitada solo por el interés general que se expresa a través de las leyes. Al someterse a las leyes que emanan de ellos mismos como parte constitutiva del soberano, las personas aceptan restricciones a su libertad privada en aras de garantizar el mismo esquema de libertades básicas iguales también para los demás, a través del carácter coercitivo del derecho.

Este punto resulta interesante, porque en Rousseau ya aparece el problema de la relación entre coerción jurídica, libertades individuales y legitimidad, que posteriormente retomarán varios pensadores como Kant, Rawls o Habermas.

De esta manera, la relación entre coerción y legitimidad se expresa mediante la reciprocidad entre autonomía privada y autonomía pública. La reciprocidad en las relaciones socio-políticas lleva al ginebrino a presentar como condiciones necesarias para la libertad, primero, el reconocimiento de derechos individuales como el derecho a la propiedad y el derecho a la libertad de conciencia y de expresión. Estos derechos imponen a su vez deberes correlativos que son regulados a través del entramado institucional de la sociedad. En segundo lugar, la necesidad del consentimiento público en torno a los principios políticos que emanan de la voluntad general se debe entender como un principio procedimental de legitimación. En cuanto al derecho a la propiedad privada, Rousseau no busca eliminarla, sino regularla de forma tal que no atente

\footnotetext{
${ }^{48}$ Rousseau, J.J., Discurso sobre el origen y los fundamentos de la desigualdad entre los hombres. Discurso sobre las ciencias y las artes. Del Contrato social, p. 43.

${ }^{49}$ Cf. Constant, B., Escritos politicos, Madrid: Centro de Estudios Politicos y Constitucionales, 1989.
} 
contra el interés común mediante el control de la acumulación del capital en pocas manos ${ }^{50}$.

El consentimiento representa una versión previa de las propuestas deliberativas de la legitimidad democrática y de la validez procedimental del derecho. El principio D formulado por Habermas puede ser entendido como una versión racional-pragmática de los criterios normativos que orientan la voluntad general: "Válidas son aquellas normas (y sólo aquellas normas) a las que todos los que puedan verse afectados por ellas pudiesen prestar su asentimiento como participantes en discursos racionales" ${ }^{51}$.

El amor de sí mismo y la piedad, asociados a la perfectibilidad humana, encuentran su encarnación en las leyes legitimas a través de la capacidad para ejercer la libertad individual y perseguir los intereses particulares dentro de un sistema de cooperación social mutuamente ventajoso, y a través del reconocimiento de los demás miembros de la sociedad como miembros iguales en derechos y como capaces de actuar siguiendo el principio normativo del interés general, prescrita por la voluntad general. La libertad positiva y la libertad negativa se encuentran pues en una relación de reciprocidad.

Como se ha mencionado ya, en la sociedad civil la libertad natural se transforma en libertad civil y moral. Al final del libro primero del Contrato social, Rousseau introduce una reflexión similar en lo que respecta a la igualdad: "Terminaré este capítulo y este libro con una observación que debe servir de base a todo el sistema social; y es que en lugar de destruir la igualdad natural, el pacto fundamental sustituye, por el contrario, por una igualdad moral y legítima lo que la naturaleza había podido poner de desigualdad fisica entre los hombres, y que, pudiendo ser desiguales en fuerza o genio, se convierten en iguales por convención y derecho"52.

Sin embargo, la idea de la igualdad moral en el contrato social no se reduce a la igualdad formal propia del liberalismo clásico. La voluntad general también exige formas de distribución de la riqueza que puedan ser aceptadas por todos los miembros de la sociedad. En una nota a la cita anterior, Rousseau introduce la idea de la necesidad de la regulación de la desigualdad económica: "Bajo los malos gobiernos, esta igualdad solo es aparente e ilusoria; no sirve más que

\footnotetext{
50 Cf. Pierson, C., "Rousseau and the Paradoxes of Property", pp. 417-418.

51 Habermas, J., Facticidad y validez. Sobre el derecho y el Estado democrático de derecho en términos de la teoría del discurso, Madrid: Trotta, 2010, p. 172.

52 Rousseau, J.J., Discurso sobre el origen y los fundamentos de la desigualdad entre los hombres. Discurso sobre las ciencias y las artes. Del Contrato social, p. 48.
} 
para mantener al pobre en su miseria y al rico en su usurpación. De hecho, las leyes son siempre útiles a los que poseen y perjudiciales a los que no tienen nada: de donde se sigue que el estado social solo es ventajoso a los hombres en tanto que todos tienen algo y ninguno de ellos tiene nada en demasía"53.

En este punto aparece nuevamente la idea de la justicia como reciprocidad: solo puede ser justa una distribución de la riqueza que tenga en cuenta lo que es bueno para todos los miembros de la sociedad, y no solo bueno o ventajoso para unos pocos. Esta distribución justa implica, a su vez, la regulación de la desigualdad fisica o las desigualdades en talentos y dotaciones innatas. Esta idea parece haber inspirado el principio de diferencia de la teoría de la justicia de Rawls, quien considera que en la sociedad hay tres tipos diferentes de contingencias que deben ser reguladas institucionalmente: la clase social de origen, las dotaciones innatas, y la buena o mala suerte que pueden tener los individuos a lo largo del desarrollo de sus vidas como miembros de un sistema de cooperación social a lo largo del tiempo ${ }^{54}$. Hasta el momento se ha dicho que Rousseau defiende la libertad y la igualdad como los dos mayores bienes de la sociedad; por tal motivo, deben ser el objetivo principal de toda legislación. $\mathrm{El}$ ginebrino sabía que el reconocimiento puramente formal de las libertades básicas no asegura que las personas puedan realmente ejercer los derechos asociados a estas. Es necesaria la justificación de la distribución a través de un principio de proporcionalidad. En palabras del ginebrino, la igualdad debe entenderse de la siguiente forma: "Ya he dicho lo que es la libertad civil; respecto a la igualdad, no hay que entender por esta palabra que los grados de poder y riqueza sea absolutamente los mismos, sino que, en cuanto el poder, éste quede por encima de toda violencia y nunca se ejerza sino en virtud del rango y de las leyes, y en cuanto a la riqueza, que ningún ciudadano sea suficientemente opulento como para comprar a otro, ni ninguno tan pobre como para ser constreñido a venderse..." ${ }^{55}$.

Si bien Rousseau no establece el punto en el cual las personas no se verian obligadas a venderse, sí podemos distinguir un principio de proporcionalidad en el acceso a la riqueza. Si la cooperación social es mutuamente ventajosa, la acumulación de capital en pocas manos no puede ser la regla que determine

\footnotetext{
53 Ibid., p. 318.

54 Rawls, J., Teoría de la justicia, Ciudad de México: Fondo de Cultura Económica, 2006, pp. 17-18.

${ }_{55}$ Rousseau, J.J., Discurso sobre el origen y los fundamentos de la desigualdad entre los hombres. Discurso sobre las ciencias y las artes. Del Contrato social, p. 76.
} 
los fines de la acción política. Esto implica que Rousseau concibe la posibilidad de la regulación de la propiedad privada a través de medidas fiscales e institucionales. La desigualdad en lo que respecta a ingreso y riqueza constituye un obstáculo para el desarrollo de las condiciones humanas de convivencia, primero porque sitúa a las personas en condiciones de dependencia económica, lo que les imposibilita ser libres; y segundo, el monopolio económico genera necesariamente monopolios politicos, asegurando el poder para aquellos que poseen la mayor cantidad de riquezas, creando asi una desigualdad política injustificable en términos de la teoría política del ginebrino.

En lo concerniente al gobierno y a las instituciones politico-administrativas del poder público, Rousseau considera que este debe ser entendido como la fuerza que ejecuta los dictados de la voluntad general; en otras palabras, el gobierno es el encargado de la administración de las leyes y de la república. Este cuerpo siempre debe actuar bajo los dictámenes de la voluntad general, para que sus decisiones y acciones puedan ser consideradas como legítimas. El gobierno está sujetado, pues, al soberano que, como ya se había mencionado, está conformado por cada uno de los miembros de la sociedad. Los gobernantes son entonces los delegados del pueblo para la administración política teniendo siempre en cuenta el interés general. Los problemas centrales para Rousseau en lo que respecta a la forma de gobierno de una sociedad justa giran en torno a la relación existente entre efectividad y legitimidad y la participación y deliberación como formas para la conservación del poder soberano por parte de los ciudadanos. En este sentido, Rousseau reconoce la importancia de la burocracia en el desarrollo del Estado moderno, fenómeno histórico que él está presenciando, pero, al mismo tiempo, identifica la necesidad de la participación ciudadana en la toma de decisiones como criterio básico de toda posible legitimidad politica y forma de control del poder político, en el marco normativo de la libertad y la igualdad como aquello que define los intereses fundamentales de los seres humanos.

Así, para el ginebrino, la mejor forma de gobierno exige la sujeción de las instituciones administrativas a los principios politicos de la sociedad, de forma tal que los puestos y cargos públicos estén abiertos a todos los ciudadanos en razón de sus méritos y la necesidad normativa y concreta de la conjunción entre la libertad y la igualdad para todos los ciudadanos constituye el marco de valoración de la legitimidad. De otra forma, la idea de la democracia como la forma de gobierno legitima no tendría razón de ser, puesto que se necesita de la deliberación y participación de los ciudadanos en las asambleas previstas 
por la ley para así poder ejercer una veeduría constante sobre las instituciones de gobierno.

Este último asunto introduce la idea del pluralismo en Rousseau. La deliberación necesariamente exige el reconocimiento de una pluralidad de visiones al interior de la sociedad. Por eso, la voluntad general debe entenderse como la suma de las pequeñas diferencias: "Si los ciudadanos no tuvieran comunicación alguna entre sí cuando el pueblo, suficientemente informado, delibera, del gran número de las pequeñas diferencias resultaría siempre la voluntad general, y la deliberación sería siempre buena"56. La diferencia es defendida por Rousseau, en contra de algunas interpretaciones que sostienen que la voluntad general vuelve homogénea a toda la población y anula la individualidad ${ }^{57}$. Este reconocimiento de la pluralidad conlleva la idea de la participación política por parte de los ciudadanos. No basta con haber sentado los principios constitucionales ni con haber elegido a los funcionarios públicos. El pueblo debe reunirse en asambleas periódicas o extraordinarias según lo exija la situación. Esta es la única forma en que se puede conservar la justicia de las acciones políticas: mediante la deliberación y la participación políticas, el pueblo puede conservar su autoridad soberana ejerciendo una vigilancia sobre la legitimidad de los actos de los gobernantes. La indiferencia política es el mayor peligro para toda sociedad que quiere basar sus relaciones sociales bajo principios de justicia.

Tan pronto como los ciudadanos se alejan de la esfera pública y se retiran a sus vidas privadas interesados solamente en sus asuntos particulares, aparecen la tiranía y el despotismo. El ejercicio del poder político no se puede entender como la dominación de una parte sobre la otra, o de una mayoría de votantes que ha salido victoriosa en las elecciones sobre la minoría perdedora. El ejercicio del poder político se entiende aquí como la deliberación y participación de todos los ciudadanos, como miembros del pueblo y guiados por la voluntad general, en los procesos de toma de decisiones. La exclusión o limitación de esta actividad conllevaría consigo el despotismo de unos cuantos sobre el resto, o la imposición de la voluntad de todos, entendida como la agregación de preferencias particulares, sobre el cuerpo colectivo y los intereses generales ${ }^{58}$.

\footnotetext{
56 Ibid., p. 53.

57 Cf. Berlin, I., La traición de la libertad. Seis enemigos de la libertad humana, pp. 70-73.

58 Cf. Rousseau, J.J., Discurso sobre el origen y los fundamentos de la desigualdad entre los hombres. Discurso sobre las ciencias y las artes. Del Contrato social, p. 52.
} 


\section{Conclusiones: el legado de Rousseau}

¿Qué enseñanza nos queda del pensamiento político de Rousseau? Esta pregunta cobra mayor relevancia en el contexto de la posdemocracia, momento histórico en el cual los grandes capitales e intereses económicos han terminado por mercantilizar la ciudadanía y obstaculizar la participación política de los sectores más vulnerables ${ }^{59}$. Este artículo ha mostrado que el legado rousseauniano se puede observar en su concepto de democracia. Esta no puede entenderse sin la libertad positiva y negativa, ni sin la igualdad social y politica de sus ciudadanos. La libertad negativa exige el reconocimiento de la esfera privada de los individuos y sus derechos civiles que les garantizan libertades individuales básicas. Las libertades positivas de participación politica incluyen la capacidad de los ciudadanos de ejercer una veeduría constante sobre las decisiones politicas y su conformidad con los intereses generales de las personas. La igualdad es indispensable a su vez porque sin esta no puede haber libertad. Sin igualdad social, las libertades básicas serian solo formales y sin igualdad política la participación sería impracticable.

La deliberación exige un consenso sobre los principios básicos de la sociedad y la sujeción de las instituciones políticas a la consecución del bien común. Ese es pues el legado rousseauniano: libertad, igualdad y deliberación como condiciones esenciales para las prácticas democráticas. Sin embargo, pareciera ser que estas están ausentes de las prácticas politicas de muchas de las sociedades que actualmente se precian de ser democráticas. En este sentido, la teoría política del ginebrino permite resaltar tres aspectos importantes en el contexto de la hegemonía de la democracia representativa liberal.

En primer lugar, Rousseau nos permite ir más allá de la idea de acción politica limitada a elecciones y contiendas partidistas propio de los regimenes democráticos contemporáneos. Esto no quiere decir que el ginebrino se oponga al voto, quiere decir que la acción política de los ciudadanos no puede reducirse a este mecanismo; por esta razón, para él, los ingleses no son verdaderamente libres, a pesar de contar con el derecho al voto ${ }^{60}$. Solo una ciudadanía activa, que participa en la toma de decisiones politicas, orientados por los intereses generales -lo que es bueno para todos y no solo para algunos- puede constituir la base de una democracia legítima.

\footnotetext{
59 Crouch, C., Post-Democracy, Cambridge: Polity Press, 2007, p. 80.

${ }^{60}$ Cf. Rousseau, J.J., Discurso sobre el origen y los fundamentos de la desigualdad entre los hombres. Discurso sobre las ciencias y las artes. Del Contrato social, p. 120.
} 
En segundo lugar, Rousseau nos deja como herencia el llamado a estar siempre vigilantes con el poder económico y las desigualdades. Como se ha mencionado, para el autor un pueblo plagado por grandes desigualdades sociales no puede ser nunca realmente libre. Así, la libertad se encuentra intrínsecamente unida a la igualdad económica, moral y política, que exige una regulación y control de la acumulación del capital y la riqueza en pocas manos. Solo bajo condiciones de reciprocidad en los términos de cooperación y producción, puede haber democracia.

Por último, como el primer crítico moderno de la modernidad, Rousseau nos invita con su teoría politica a tomar partido en contra de toda forma de despotismo, incluso el ilustrado, de manera tal que la crítica a los centros de poder y a las diferentes condiciones de opresión, dominación y exclusión nunca termine.

Recibido: 06/08/2020

Aceptado: 12/02/2021

\section{Bibliografia}

Althusser, L., Lessons on Rousseau, Londres: Verso, 2019.

Anderson, P., El Estado absolutista, Madrid: Siglo XXI, 2016.

Arango, I.D., Críticos y lectores de Rousseau, Medellín: Editorial Universidad de Antioquia, 2006.

Berlin, I., La traición de la libertad. Seis enemigos de la libertad humana, Ciudad de México: Fondo de Cultura Económica, 2004.

Berlin, I., Dos conceptos de libertad. El fin justifica los medios. Mi trayectoria intelectual, Madrid: Alianza Editorial, 2014.

Cohen, J., Rousseau. A Free Community of Equals, Oxford: Ocford University Press, 2010. https://doi.org/10.1093/acprof:oso/9780199581498.003.0002

Constant, B., Escritos políticos, Madrid: Centro de Estudios Políticos y Constitucionales, 1989.

Crouch, C., Post-Democracy, Cambridge: Polity Press, 2007.

116 De Dijn, A., "Rousseau and Republicanism", en: Political Theory, v. XLVI, 1 (2018), pp. 59-80. https://doi.org/10.1525/abt.2018.80.1.59

Derathé, R., Rousseau et la Science Politique de son temps, París: Libraire philosophique J. Vrain, 1995.

Garrard, G., "Rousseau: Happiness and Human Nature", en: Political Studies, v. LXII (2014), pp. 70-82. https://doi.org/10.1111/j.1467-9248.2012.00990.x 
Habermas, J., Facticidad y validez. Sobre el derecho y el Estado democrático de derecho en términos de la teoría del discurso, Madrid: Trotta, 2010.

Hardt, M. y A. Negri, Commonwealth, Cambridge/Londres: Harvard University Press, 2009. https://doi.org/10.2307/j.ctvjsf48h

Kersting, W., Jean Jacques Rousseaus “Gesellschafts Vertrag”, Darmstad: Wissenschaftliche Buchgesellschaft, 2002.

Locke, J., Segundo tratado sobre el gobierno civil, Madrid: Alianza editorial, 2010.

Pierson, C., "Rousseau and the Paradoxes of Property", en: European Journal of Political Theory, v. XII, 4 (2013), pp. 409-424. https://doi.org/ 10.1177/1474885112449586

Rawls, J., Teoria de la justicia, Ciudad de México: Fondo de Cultura Económica, 2006.

Rousseau, J.J., Discurso sobre el origen y los fundamentos de la desigualdad entre los hombres. Discurso sobre las ciencias y las artes. Del Contrato social, Madrid: Alianza Editorial, 2008.

Rubio, J., "Rousseau y Kant: una relación proteica", en: Revista de Estudios Politicos. 133 (2006), pp. 9-37.

Schwartzberg, M., "Rousseau on Fundamental Law", en: Political Studies, v. LI (2003), pp. 387-403. https://doi.org/10.1111/1467-9248.00430

Thomä, D., Puer Robustus. Una filosofía del perturbador, Barcelona: Herder, 2018.

Thomas, P., "Among Prelates and Primates. From Darwin to Rousseau", en: Political Theory, v. XXXVII, 34 (2009), pp. 455-480. https://doi.org/10.1177/0090591709335200 